\title{
10 Dating violence and suicidal behavior in adolescents
}

\author{
Kristin Holmes and Leo Sher
}

The aim of this chapter is to assess the possible consequences of adolescent physical, emotional and sexual dating violence through a review of the literature on the topic. An electronic search of major biomedical bibliographic databases (Pubmed, ISI, Psyc INFO) was used to retrieve articles providing information on the prevalence rates, risk factors, associated consequences and possible preventive measures for adolescent dating violence across different populations. Currently, there have been few longitudinal studies conducted to identify potential risk factors for entering a violent dating relationship in adolescents. Risky behaviors such as earlier sexual intercourse may predispose someone for victimization. Dating violence itself is also a predictor of future dating violence. Adolescent dating violence was associated with an increase in other violence-related behaviors, substance use, depression, poorer educational outcomes, post-traumatic stress, unhealthy weight control, and risky sexual behavior. The association between adolescent dating violence and an increase in suicidal behavior is a major public health concern. Future research should focus on longitudinal studies so that a causal relationship between dating violence and suicidality may be better understood.

\subsection{Introduction}

Approximately 9\% of adolescents are victims of dating violence (1). As this review of the current literature reveals, dating violence has numerous physical and psychological implications, the most serious of which is suicidality. The prevalence of dating violence in adolescent relationships and its consequences (including suicidality) is discussed and purported risk factors for its occurrence are reviewed. Additionally, limitations of the current research are examined and directions for future research and prevention are proposed.

According to the Center for Disease Control and Prevention (CDC), adolescent partner violence can be characterized along four dimensions: emotional, physical, sexual, and stalking (1). Each categorization consists of a number of violent behaviors, which range in severity. Emotional dating violence includes verbal abuse, and jealous or controlling behaviors such as preventing the partner from talking to members of the opposite sex, checking text messages or emails, or not allowing the partner to keep in touch with members of his or her friends and family. Physical dating violence consists of everything from pushing or restraining to hitting, punching, choking, or the use of a weapon against one's partner. Sexual dating violence includes uncomfortable remarks as well as any unwanted sexual experiences. Stalking includes threats or patterns of harassment that are used to instill fear in the victim (1). 


\subsubsection{Literature search}

An electronic search of the major biomedical bibliographic databases (Pubmed, ISI, PsycINFO) was conducted to retrieve articles published between the years 2000 and 2012 describing the social, epidemiological and clinical characteristics of dating violence and its relationship to suicidal behavior in adolescents. Key words for the search included "adolescent," "teen," "dating violence," "victimization," "abuse," "suicide," and "suicidal behavior."

\subsection{Findings}

Upon analysis of the prevalence of dating violence in adolescents, it becomes apparent that this is a widespread problem among youth. Across both genders, approximately $9-17 \%$ of adolescents report physical dating violence $(2,3)$. The variation in prevalence rates may be attributable to differences in sample populations. Among New York City youth, $10.6 \%$ of females and $9.5 \%$ of males reported physical dating violence (4). Similar results were found in a nationally representative sample of boys with 9.13\% reporting physical dating violence (5).

A study focusing on Black and Hispanic youth found that approximately $10 \%$ of youths reported dating violence victimization in the past year (6). In a survey study of Latino youths aged 11-13 residing in suburban Washington DC, prevalence of dating violence was $14.4 \%$ for girls and $12.9 \%$ for boys (13.5\% across the sample) (7). Prevalence of dating violence among adolescent boys was highest for those who classified themselves as black or ethnically "other” (5). These slightly higher rates of dating violence among minorities may be due to socio-economic status or cultural differences.

In a study of New York City youth, 9.6\% of females and 5.4\% of males reported a lifetime history of sexual assault (4). 13.2\% of adolescents in a large survey of students in grades 7-12 reported unwanted sexual experiences perpetrated by a peer (2). A study focusing on Black and Hispanic youth found that approximately one in 10 youths reported dating violence victimization in the past year and 1 in 10 girls reported a history of sexual assault in her lifetime (6). Analysis of the 1997 and 1999 Massachusetts Youth Risk Behavior Surveys revealed that about one in five female adolescents had been a victim of physical and/or sexual dating violence (20.2\% in 1997 and $18.0 \%$ in 1999) (8). Another large survey-based study found that $24.6 \%$ of youths in grades 7-12 reported at least one of these forms of victimization (2).

Although both boys and girls experience dating violence victimization, in a study of 190 high school students, girls reported higher levels of severity and frequency of dating violence (9). In concurrence, another study found that males are more apt to engage in severe forms of physical aggression ( $4.6 \%$ vs. $2.0 \%$ ) that result in serious medical consequences for the victim (10). Females, however, reported a significantly higher percentage of verbal aggression than males (10). 


\subsubsection{Risk factors}

To date, most research on adolescent dating violence has been cross-sectional in nature. Thus, while many risk factors for engaging in adolescent dating violence have been proposed (i.e., exposure to domestic violence in the family of origin, low socioeconomic status, etc.), they have yet to be validated scientifically. Similarly, a causal relationship between mental health symptoms such as depression, anxiety, or suicidality and dating violence cannot be established. There have been a few longitudinal studies, however, that provide some insight into precursors for and consequences of adolescent dating violence.

One such study proposed several potential risk factors for dating violence (11). This study followed girls dating in grade 9 in an attempt to predict profiles of dating relationships in grade 11 based on the girls' relationships with families and peers. They found that an adolescent girl's involvement in a mutually violent dating relationship in grade 11 could be facilitated by several risk factors (i.e., delinquency, parental rejection, and sexual harassment perpetration) evident during grade 9. Moreover, girls in grade 11 who reported being solely victims in violent dating relationships had higher rates of sexual intercourse in grade 9 (11). This implies that early relationships may predispose a girl to future victimization during later adolescence. Sexual relationships at this young of an age may directly place girls at more risk for later physical violence. Or, perhaps these girls have some personality characteristics that lead them to relationships with a certain aggressive type of male or to engage in riskier behaviors that consequently make them more vulnerable to victimization.

Dating violence in itself is a risk factor for further dating violence. One study found that $8 \%$ of boys and $15 \%$ of girls were persistently aggressive with the same partner over a period of 3 months. In a year-long longitudinal study of dating aggression in Canadian high-school students, $13 \%$ of participants reported recurrent dating aggression across two different relationships (12). Of the remaining participants, 55\% reported no dating aggression at either point and 32\% reported dating aggression in one of their relationships during this time period. This study was unique as it was the first to study recurrent aggression across multiple dating relationships. Different patterns of aggression emerged from these groups. For those who reported dating violence in only one relationship, frequency of dating aggression was low (most reporting that the acts occurred "rarely") and the severity was reported as being minor (pushing, grabbing, or shoving). For those who reported aggression in two relationships, the frequency of aggression was higher at the second data collection point. Also, most adolescents reported that aggression occurred in both directions, with each partner acting as a victim and a perpetrator (12). Another study also found that victimization in a dating relationship predicted perpetration (13). Therefore, being a victim of dating violence not only puts an adolescent at risk for further victimization, but also becomes a risk factor for perpetration. 
These findings suggest that patterns emerge in early relationships that make an adolescent vulnerable to victimization and/or perpetration of physical dating violence. If these risk factors are identified early on, later dating violence could be prevented. Perpetration of physical violence can be predicted by previous victimization and engagement in psychological aggression (i.e., verbal aggression, jealous behavior, and controlling behavior) (14). Due to this "circle-of-violence" in which the victim evolves into the perpetrator, it is especially important to intervene at early stages and break this cycle before violent patterns are materialized.

\subsubsection{Psychiatric and social consequences of dating violence}

Cross-sectional studies found that dating violence was associated with an increase in other risk behaviors and mental health consequences such as violence-related behaviors, substance use, depression and suicidal ideation (3). Dating violence was also associated with poorer educational outcomes (2), post-traumatic stress (9), and unhealthy weight control including diet pill use, laxative use and vomiting (8). In a nationally representative sample, risky sexual behavior was also associated with physical dating violence $(5,8)$. More specifically, those boys who reported two or more sexual partners in the past 3 months, compared with those who reported none, were over three times more likely to report violence (5).

Two different US surveys of risk behavior in youths found an association between drinking alcohol, smoking cigarettes and using cocaine with dating violence $(5,8)$. In a study of Latino early adolescents, girls who binge drank were 27 times more likely to report dating violence (7). The association between current alcohol consumption and dating violence was also seen among male high-school students in central Thailand further proving that this finding extends to many different cultures (15).

\subsubsection{Dating violence and suicidality}

Suicidality is a serious consequence of adolescent dating violence. The adolescent suicide attempt rate is high, with a 2005 New York City survey finding that $11.7 \%$ of female and $7.2 \%$ of male adolescents have made one or more suicide attempts (4). Dating violence victimization may be a significant risk factor for adolescent suicidality as they were found to be associated in several studies $(6,16)$. In one study of adolescent abortion patients, dating violence was related to severity of suicidal thinking; and, as the severity of the adolescent females' general problems with aggressive behavior rose, the magnitude of the relationship between dating violence and severity of suicidal thinking increased (17). This suggests that there may be personality traits or psychiatric illnesses that mediate the relationship between dating violence and suicidality. In another study, female Asian-American and Pacific Islander youth 
were significantly more likely to consider suicide and to have made a suicide attempt than males (18). Therefore, dating violence victimization may be a greater hazard for female adolescents than male. Even though dating violence can increase the frequency and severity of suicidal thoughts and increase the risk of making an attempt, research on this topic is sparse. Additional research with a broader sample would be beneficial to the development of prevention initiatives for both adolescent dating violence and suicidality.

\subsection{Discussion}

There are many theoretical mechanisms which may account for an increase in suicidality resulting from dating violence. While dating violence can directly increase the risk for suicidality in the victim, it more often leads to other health consequences, which in turn bolster suicidal ideation and behavior. These consequences act as mediators, as they facilitate the risk for suicidality, due indirectly to engagement in dating violence.

One such mediator could be the multitude of negative mental health consequences that were discussed earlier such as depression, anxiety, post-traumatic stress, substance use, or disordered eating patterns. These mental health outcomes in turn increase the risk for suicidal thoughts or behaviors in the victim. Another possible mediator, which requires further study, is the medical consequences associated with dating violence. Some medical consequences of dating violence such as bruises, broken bones, and cuts have been discussed. As these adolescents grow older, the medical consequences may become even more severe leading to injuries requiring major medical stabilization or a lifetime of disability such as traumatic brain injuries (TBIs). It is very likely that these medical consequences can lead to an increase in suicidal behavior. For example, multiple lines of evidence suggest that TBIs are associated with suicide attempts and suicides $(19,20)$.

There may also be more complicated mechanisms facilitating suicidality in this population. Psychiatric illnesses such as depression or substance use may predispose an adolescent to violent dating relationships. Therefore, the adolescent is more likely to have an increase in suicidality after involvement in dating violence because of the pre-existing mental health issue. Suicidal behavior may have a possible genetic component that may be mediated by familial transmission of impulsive aggression (21).

Finding a method to reduce suicidality in adolescents is of extreme importance. Dating violence is one reason an adolescent may suffer from suicidal thoughts and have made one or more suicide attempts. Identifying at-risk youth should be the first step towards prevention of both dating violence and suicidality. Early detection of risk factors for suicidal behavior may help identify families that are at risk (21). This is important because the family unit is an integral part of the adolescents' development and family connectedness is inversely related to dating violence (3). If an at-risk 
family is identified early-on, perhaps the family can receive the resources they need to promote family connectedness and prevent negative developmental outcomes such as depression, substance abuse, dating violence and suicidality.

Educating those families and youth that are at risk about dating violence should be the next step after identification towards prevention. Media may be a beneficial means to educating adolescents because teens tend to be influenced by what they see in the media and pop-culture. Informative and positive media campaigns may reduce the negative effects of other forms of mass media that may instigate teen dating violence or other risky behaviors (22). Youth are also influenced by the environment in which they live. Therefore, attempts at educating youth should extend past the use of media to include the family, schools and community programs. A multi-dimensional approach between pediatricians, families, schools, and communities would be the most effective strategy towards prevention of suicide attempts in adolescents (6). This multi-dimensional approach should include as stated, education, but also include attempts to get youth involved in positive programs that will make them feel connected to the community, enhance their sense of self and reduce involvement in risky behaviors and past-times. Sports and art, music, or drama programs are examples of some positive activities that families, schools, and communities can offer youth to prevent negative behaviors that may lead to dating violence and suicidality.

Future research on this very important topic should focus on longitudinal studies so that a causal relationship between dating violence and suicidality might be better understood. Mediating factors (i.e., medical problems, depression, and substance use) should also be examined due to their role in adolescent suicidality and violence. Identifying all risk factors for dating violence and suicidality is essential for implementing effective intervention strategies.

\section{References}

1. Centers for Disease Control and Prevention. Youth risk behavior surveillance - United States, 2011. MMWR, Surveillance Summaries 2012;61(SS-4):1-162.

2. Banyard VL, Cross C. Consequences of teen dating violence: Understanding intervening variables in ecological context. Violence Against Women 2008;14(9):998-1013.

3. Howard DE, Beck K, Kerr MH, Shattuck T. Psychosocial correlates of dating violence victimization among Latino youth. Adolescence 2005;40(158):319-31.

4. Olshen E, McVeigh KH, Wunsch-Hitzig RA, Rickert VI. Dating violence, sexual assault, and suicide attempts among urban teenagers. Arch Pediatr Adolesc Med 2007;161:539-45.

5. Howard DE, Wang MQ. Psychosocial factors associated with adolescent boys' reports of dating violence. Adolescence 2003;38(151):519-33.

6. Bonomi AE, Kelleher K. Dating violence, sexual assault, and suicide attempts among minority adolescents ending the silence. Arch Pediatr Adolesc Med 2007;161:609-10.

7. Yan FA, Howard DE, Beck K, Shattuck T, Hallmark-Kerr M. Psychosocial correlates of physical dating violence victimization among Latino early adolescents. J Interpers Violence 2010;25(5):808-31. 
8. Silverman JG, Raj A, Mucci LA, Hathaway JE. Dating violence against adolescent girls and associated substance use, unhealthy weight control, sexual risk behavior, pregnancy, and suicidality. J Am Med Assoc 2001;286(5):572-9.

9. Callahan MR, Tolman RM, Saunders DG. Adolescent dating violence victimization and psychological well-being. J Adolesc Res 2003;18(6):664-81.

10. Munoz-Rivas MJ, Grana JL, O'Leary KD, Gonzalez MP. Aggression in adolescent dating relationships: Prevalence, justification, and health consequences. J Adolesc Health 2007;40:298-304.

11. Chiodo D, Crooks CV, Wolfe DA, Mclsaac C, Hughes R, Jaffe PG. Longitudinal prediction and concurrent functioning of adolescent girls demonstrating various profiles of dating violence and victimization. Prev Sci 2011;epub. doi: 10.1007/s11121-011-0236-3.

12. Williams TS, Connolly J, Pepler D, Craig W, Laporte L. Risk models of dating aggression across different adolescent relationships: A developmental psychopathology approach. J Consult Clin Psychol 2008;76(4):622-32.

13. Nocentini A, Menesini E, Pastorelli C. Physical dating aggression growth during adolescence. J Abnorm Child Psychol 2010;38:353-65.

14. O'Leary KD, Slep AM. A dyadic longitudinal model of adolescent dating aggression. J Clin Child Adolesc Psychol 2003;32(3):314-27.

15. Chaveepojnkamjorn W, Pichainarong N. Current drinking and health-risk behaviors among male high school students in central Thailand. BMC Public Health 2011;11:233.

16. Ackard DM, Eisenberg ME, Neumark-Sztainer D. Long-term impact of adolescent dating violence on the behavioral and psychological health of male and female youth. J Pediatr 2007;(151): 476-81.

17. Ely GE, Nugent WR, Cerel J, Vimbba M. The relationship between suicidal thinking and dating violence in a sample of adolescent abortion patients. Crisis 2011;32(5):246-53.

18. Else IRN, Goebert DA, Bell CK, Carlton B, Fukuda M. The relationship between violence and youth suicide indicators among Asian American and Pacific Islander youth. Aggression Violent Behav 2009;14:470-7.

19. Brenner LA, Ignacio RV, Blow FC. Suicide and traumatic brain injury among individuals seeking Veterans Health Administration services. J Head Trauma Rehabil 2011;26(4):257-64.

20. Wasserman L, Shaw T, Vu M, Ko C, Bollegala D, Bhalerao S. An overview of traumatic brain injury and suicide. Brain Inj 2008;22(11):811-9.

21. Bursztein C, Apter A. Adolescent suicide. Curr Opin Psychiatry 2008;22:1-6.

22. Manganello JA. Teens, dating violence, and media use: A review of the literature and conceptual model for future research. Trauma Violence Abuse 2008;9(1):3-18. 
